\title{
Perspective
}

PERSPECTIVE Actualité en histoire de l'art

4 | 2006

La monographie d'artiste

\section{Carlo Crivelli : style ou iconographie}

\section{Thomas Golsenne}

\section{(2) OpenEdition}

\section{Journals}

Édition électronique

URL : http://journals.openedition.org/perspective/4224

DOI : $10.4000 /$ perspective. 4224

ISSN : 2269-7721

Éditeur

Institut national d'histoire de l'art

Édition imprimée

Date de publication : 31 décembre 2006

Pagination : 620-625

ISSN : 1777-7852

\section{Référence électronique}

Thomas Golsenne, «Carlo Crivelli : style ou iconographie », Perspective [En ligne], 4 | 2006, mis en ligne le 31 mars 2018, consulté le 01 octobre 2020. URL : http://journals.openedition.org/perspective/4224 ; DOI : https://doi.org/10.4000/perspective.4224 
18. Anna Maria Ambrosini Massari, « Ricci, Maggiori, Gentile : la nascita della storia dell'arte nelle Marche e un disegno ", dans Cecilia Prete éd., Gentile da Fabriano, "Magister Magistrorum", (colloque, Fabriano, 2005), Senigallia, 2006, p. 129-146. L'œuvre ne fut en possession de la famille Ottoni de Matelica que dans un second temps : Alberto Bufali, "Nuovi documenti per Carlo Crivelli e Luca Signorelli ed una ipotesi per gli inizi di Gentile da Fabriano ", dans Pierluigi Moriconi éd., Storie da un archivio : frequentazioni, vicende e ricerche negli archivi camerinesi (colloque, Camerino, 2006), Camerino, 2006, p. 43-50 et 62.

19. Cette proposition a été développée à l'occasion de l'exposition de Fabriano : Matteo Ceriana, Emanuela Daffra, " Il Polittico di Valle Romita : la sua storia nel museo ", dans Matteo Ceriana, Emanuela Daffra éd., Gentile da Fabriano. Il Polittico di Valle Romita, (cat. expo., Milan, Pinacoteca di Brera, 1993), Milan, 1993, p. 27-30 ; Eadem, dans Laureati, Mochi Onori, 2006, cité n. 14, p. 140-143.

20. Marcelli, 2005, cité n. 8, p. 54.

21. Pour une synthèse sur ce sujet, voir Frosinini, 2006, cité n. 15 , p. 20-21.

22. Miklós Boskovits, dans Luciano Bellosi éd., Masaccio e le origini del Rinascimento, (cat. expo., San Giovanni Valdarno, Casa Masaccio, 2002), Milan, 2002, p. 160-163. Alessandro Cecchi, dans Laureati, Mochi Onori, 2006, cité n. 14, p. 256261, ignore cette contribution et affirme qu'il n'y a aucune trace de l'image de saint Bernard, mais, paradoxalement, l'intitulé de la notice précise qu'il s'agit de saint Nicolas de Bari et saint Bernard.

23. De Marchi, 1992, cité n. 1, p. 49-51.

24. Laura Laureati, "Gentile, scomparso, torna a Fabriano dopo qualche secolo. Una breve storia della dispersione ottocentesca di alcune opere di Gentile e della loro parziale ricomposizione in occasione della mostra ", dans Laureati, Mochi Onori, 2006, cité n. 14, p. 53-59 ; Elisabetta Federici, " Gentile da Fabriano nei taccuini di un connoisseur inglese : Charles Lock Eastlake ", dans De Marchi, Laureati, Mochi Onori, 2006, cité n. 5, p. 143-153. Sur les déplacements des œuvres de Gentile da Fabriano au $\mathrm{Xv}^{\mathrm{e}}$ siècle, le travail de Stefano L'Occaso est essentiel : Fonti archivistiche per le arti a Mantova tra Mediovo e Rinascimento (1382-1459), Mantoue, 2005, p. 216, 222.

25. Voir les essais de Nicola Ann MacGregor et Sandra Freschi, dans Alessandro Cecchi éd., Gentile da Fabriano agli Uffizi, Milan, 2005, p. 173-180 et p. 181-186; ceux du volume de Ciatti, Frosinini, 2006, cité n. 15 ; voir aussi Roberto Bellucci, Cecilia Frosinini, «Tecnica e stile : appunti su Gentile da Fabriano ", dans De Marchi, Laureati, Mochi Onori, 2006, cité n. 5, p. 55-65, et les contributions ajoutées au dossier de Ceriana, Daffra, cité n. 19.

26. Pietro Zampetti, Giampiero Donnini, Gentile e $i$ pittori di Fabriano, Florence, 1992.

27. Keith Christiansen, "L'Adorazione dei Magi di Gentile da Fabriano », dans Cecchi, 2005, cité n. 25, p. 11-40.

28. Christiansen, 1982, cité n. 3, p. 37 : «... remains [...] the most remarkable and precocious painting executed up to that moment in the fifteenth century ".
29. Ibidem, p. 64 : "The fact remains that the Adoration of the Magi is a revolutionary work not because of the social standing of its artist or its relation to the hypothetical taste of Palla Strozzi, but because it expounds a new relation between painting and experience ".

30. De Marchi, 1992, cité n. 1, p. 9.

31. Laureati, Mochi Onori, 2006, cité n. 14; De Marchi, Laureati, Mochi Onori, 2006, cité n. 5.

32. Maria Rita Silvestrelli, dans Laureati, Mochi Onori, 2006, cité n. 14, p. 118-120.

33. Voir note 19.

34. Andrea De Marchi, "Gentile e la sua bottega ", dans De Marchi, Laureati, Mochi Onori, 2006, cité n. 5, p. 9-53.

35. Vasari, 1568, cité n. 7, p. 51.

36. Andrea De Marchi, " Gentile da Fabriano et Pisanello à Saint-Jean de Latran ", dans Dominique Cordellier, Bernadette Py éd., Pisanello, (colloque, Paris, 1996), Paris, 1998, vol. 1, p. 161-213.

37. Daniele Benati, dans Jadranka Bentini, Gian Piero Cammarota, Daniela Scaglietti Kelescian éd., Pinacoteca Nazionale di Bologna. Catalogo generale. 1. Dal Duecento a Francesco Francia, Venezia, 2004, p. 180-181.

Mauro Minardi, Università degli Studi della Basilicata

mauromi@katamail.com

\section{Carlo Crivelli : style ou iconographie Thomas Golsenne}

La bibliographie du peintre d'origine vénitienne Carlo Crivelli (v. 1435 - v. 1494) commence par une lacune. Crivelli fait en effet partie des rares grands artistes de la Renaissance absents des Vite de Vasari. Les raisons de cette absence sont aisées à imaginer. La Marche d'Ancône, où travaillait Crivelli, ne comptait pas à l'époque de Vasari de grands centres artistiques, et l'art toscan - le meilleur, selon Vasari - avait peu pénétré dans le sud de la région, où Crivelli opérait. En outre, le style même de Crivelli devait susciter peu d'intérêt de la part du biographe arétin : s'il avait regardé ses polyptyques, illes avait probablement considérés commelesœuvres d'un lointain disciple de Gentile da Fabriano, trop gothiques, trop décoratifs ; autrement dit, d'un peintre qui n'avait pas su prendre 
le train du progrès des arts et tournait le dos aussi bien à la modernité florentine d'un Botticelli qu'à la nouveauté vénitienne d'un Bellini.

L' « oubli » de Vasari concernant Crivelli pose un problème remarquable quand on s'intéresse au genre de la monographie dans le domaine de l'art de la Renaissance, car ce genre littéraire découle précisément du texte vasarien : une histoire générale des arts de la période conçue comme une succession généalogique de biographies, qui sont autant de monographies plus ou moins longues ${ }^{1}$. Chez Vasari, la vie et l'œuvre de l'artiste se reflètent l'une et l'autre, et la trace de cette réflexion est la maniera, ce que nous appelons le style. L'histoire de l'art à la Vasari est donc une histoire des styles, dont la plus petite unité est le catalogue monographique de chaque artiste étudié. Ainsi chaque monographie vasarienne est-elle prise dans un mouvement qui la dépasse, chaque artiste subit et produit des influences.

Une bonne partie des monographies écrites par les historiens modernes de l'art de la Renaissance s'inscrit dans ce schéma vasarien. Même si le livre fonde ses limites sur celles de la vie et du catalogue de l'artiste, et trouve son fil conducteur dans son style, il ne peut omettre le contexte artistique qui le rend possible et qui lui donne un sens historique, c'est-à-dire une place dans la généalogie inventée par Vasari. On comprend dès lors la difficulté d'écrire une monographie sur Crivelli sans pouvoir s'appuyer sur aucun repère vasarien : donner un sens à son œuvre, c'est en même temps écrire un chapitre de l'histoire de l'art du Quattrocento omis par Vasari. C'est ce à quoi la majorité des historiens de l'art se sont employés jusqu'à très récemment. Mais l'étude de la bibliographie crivellesque nous montre que le cas Crivelli peut remettre en cause beaucoup plus profondément le modèle vasarien. Le problème posé par cet artiste et ses monographies a donc une portée générale, il est emblématique des façons dont on peut écrire l'histoire de l'art du Quattrocento.

\section{Un anti-moderne du Quattrocento}

Comment intégrer l'œuvre de Crivelli dans l'histoire de l'art vasarienne sans dénaturer celleci ? À lire la bibliographie crivellesque, les historiens de l'art ont trouvé deux manières de répondre à cette question. La première est de l'intégrer au chapitre sur la peinture vénitienne. Après tout, Crivelli a signé pendant toute sa vie " de Venise ".
Telle est la démarche de Lanzi à la fin du XVIII ${ }^{\mathrm{e}}$ siècle, dans sa grande histoire de l'art de l'Italie par régions ${ }^{2}$, suivi en cela par Van Marle ou Venturi au début du $\mathrm{xx}^{\mathrm{e}}$ siècle ${ }^{3}$. L'art de Crivelli est ainsi considéré comme un exemple de style gothique vénitien tardif, représentant un courant réactionnaire, archaïque, voire décadent de la peinture vénitienne, si on le compare à celui du " moderne " Bellini. La peinture de Bellini est tournée vers l'avenir, vers Titien. Celle de Crivelli est tournée vers le passé. Cette approche assez négative a imprégné les esprits pour longtemps. Ainsi, Roberto Longhi, dans un article de 1961, donne une explication géo-culturelle à la décadence crivellesque. Il observe comment certains peintres de sa génération, bénéficiant d'une ambiance culturelle vivante, comme Mantegna à Mantoue, Tura à Ferrare ou Bellini à Venise, s'en sont bien sortis, et comment d'autres ont vu leur inspiration se ternir du fait de leur enterrement dans une région isolée et retardataire, comme Crivelli dans la province d'Ascoli Piceno ${ }^{4}$.

Le seul à cette époque à couvrir de louanges la peinture de Crivelli tout en insistant sur sa formation vénitienne est aussi l'auteur de la première monographie parue sur le peintre et il n'est pas italien. Ce n'est pas un hasard. Quand George McNeil Rushforth publie son Carlo Crivelli en $1900^{5}$, un texte enthousiaste mais érudit, il s'adresse au public qui, de toute l'Europe, aime le plus passionnément la peinture du Vénitien. Depuis la moitié du XIX ${ }^{e}$ siècle en effet, les amateurs d'art anglais et la National Gallery dépensent sans compter et sans équivalent ailleurs pour posséder des peintures de Crivelli ${ }^{6}$. Ce goût s'explique assez bien : les préraphaélites ont diffusé leur penchant pour les peintres italiens du $\mathrm{Xv}^{\mathrm{e}}$ siècle, Ruskin se fait l'apôtre du gothique (en particulier vénitien) et le mouvement Arts \& Crafts donne à l'ornement toutes ses lettres de noblesse ${ }^{7}$. La conjonction de ces trois facteurs est unique et détermine le succès de Crivelli dans l'Angleterre de Rushforth.

Celui-ci analyse avec subtilité le paradoxe du style de Crivelli, à la fois " archaïque, réactionnaire " et en même temps doté d'un "sentiment exquis et de riche effet décoratif ". Les contradictions qui parcourent l'œuvre de Crivelli (il "résume toutes les ressources de la pratique byzantine " et recherche le réalisme dans les fruits et les fleurs, il produit des polyptyques mais n'ignore pas la perspective) ne sont pas considérées par Rushforth 


\section{Carlo}

Crivelli,

Imago Pietatis

(provenant du

Polyptyque de

Montefiore),

1470-1475,

Londres, $\mathrm{Na}$ -

tional Gallery.
2. Carlo

Crivelli, Sainte

Conversation,

1487, Lisbonne, collection

privée. comme des faibles-

ses mais comme

les caractéristiques de l'originalité absolue de Crivelli. Non vasarienne dans ses choix esthétiques, la monographie de Rushforth le reste dans son plan, qui présente le catalogue

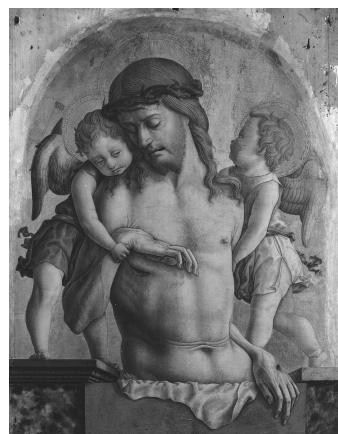
de Crivelli par ordre chronologique, depuis ses années de formation à Venise jusqu'aux œuvres de ses successeurs immédiats. Cependant cette contextualisation généalogique ne se double pas d'une comparaison synchronique. Son mérite - ou son défaut - est de ne pas comparer Crivelli à ses contemporains plus prestigieux, ce qui lui donne toutes ses chances, mais l'isole. De même d'ailleurs que le livre de Rushforth dont le goût pour le gothique décoratif est trop daté et pas assez vasarien pour pouvoir déterminer une ligne d'interprétation durable parmi les historiens de l'art de la Renaissance postérieurs.

La tendance qui a triomphé chez ces derniers, jusqu'à la monographie de Lightbown, est fidèle au point de vue vasarien et permet d'y englober Crivelli. Il suffit d'en faire le meilleur représentant d'une école régionale à laquelle Vasari n'avait pas prêté assez d'attention: la peinture de la Marche d'Ancône. Ainsi, au lieu d'être le dernier rejeton du gothique vénitien, Crivelli devient le chef de file d'une école de peinture, que les Italiens appelleront les "crivelleschi " Un premier ouvrage en ce sens est écrit par l'Allemand Franz Drey (1927) ${ }^{8}$, qui veut faire de ces peintres proches de Crivelli une école locale, à l'instar de l'école de Squarcione à Padoue. Mais en fait cette interprétation trouve ses origines chez les historiens de l'art des Marches du XVIII ${ }^{\mathrm{e}}$ siècle, qui vantent le patrimoine local, dans un esprit très vasarien de compétition. Sa version moderne est défendue, à partir de l'après-guerre, avec une ampleur inégalée, par Pietro Zampetti. Originaire d'Ancône, il va devenir, en quarante ans de carrière (depuis ses premiers travaux du début des années cinquante à la fin des années quatrevingts), le "Monsieur Crivelli » italien, en même temps qu'il aura acquis la réputation du plus grand spécialiste de l'art des Marches et une situation de quasi-monopole sur la question ${ }^{9}$. Rares sont les voix divergentes, surtout en Italie ${ }^{10}$.

\section{L'oil écrit}

Zampetti a organisé deux expositions autour de Crivelli (en 1950 et en 1961) ${ }^{11}$ et publié deux monographies sur son ouvre ${ }^{12}$ (sans compter des dizaines d'articles). Si leur impact fut si fort, c'est parce que ses écrits, notamment ses monographies, sont particulièrement efficaces. D'abord il a réussi à concilier l'inconciliable : défendre Crivelli comme un grand peintre, et rester fidèle à Vasari. Aux sources d'influence de Crivelli généralement invoquées (le gothique vénitien, l'école de Padoue), Zampetti en ajoute une inédite : celle de Bellini, son compatriote vénitien, formé lui aussi à Padoue, pour sa recherche sur la lumière et $l^{\prime}$ « humanité " de ses figures ${ }^{13}$. Si bien que l'histoire du style même de Crivelli est considérée par Zampetti comme une décadence et ses meilleures œuvres sont celles du début, notamment quand l'influence bellinienne se fait le mieux sentir, comme dans le Polyptyque de Montefiore ${ }^{14}$ (fig. 1). Crivelli n'est jamais meilleur que quand il n'est pas Crivelli...

Mais si la position de Zampetti s'est quasiment imposée en Italie comme un monopole, c'est surtout grâce à l'efficacité de son écriture elle-même. J'entends "écriture " au sens toujours opératoire (à mon avis) de Roland Barthes : l'écriture est un "acte de solidarité historique ", un engagement vis-à-vis de la société, un choix éthique ${ }^{15}$. Il y a pour Barthes une écriture marxiste, une écriture neutre. Je qualifierais celle de Zampetti de « vasarienne ". L'écriture de Zampetti a en effet elle aussi des effets éthiques, dans la mesure où l'auteur s'approprie l'artiste dont il étudie l'œuvre et en tire une autorité pas seulement intellectuelle. Il tire en effet son droit $d^{\prime}$ expertise de son " oeil " de spécialiste, qui est la forme objectivée de son point de vue subjectif sur l'œuvre. Mais cet « œil " se transforme parfois en simple argument d'autorité quand il

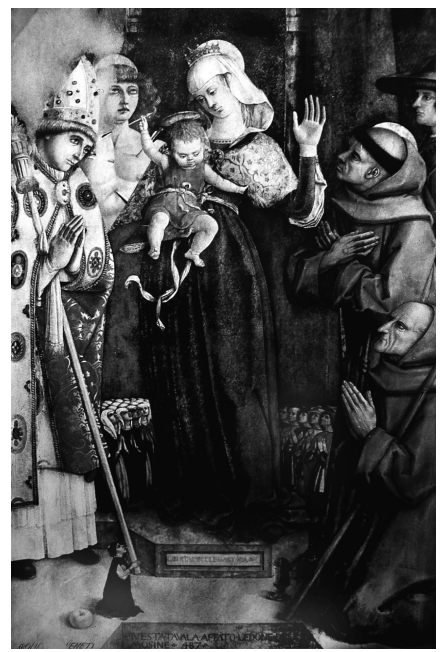


s'agit de défendre une position locale dominante. Ainsi, le plus grand mérite de Zampetti est d'avoir établi le catalogue raisonné de l'œuvre de Crivelli. Est-il pour autant définitif et totalement dénué de subjectivité ? Zampetti juge par exemple comme sa meilleure ouvre le Polyptyque de Montefiore (fig. 1), très dispersé, ni signé ni daté, mais dont il est, à ses dires, $\mathrm{l}^{\prime}$ " inventeur ${ }^{16}$. En revanche un tableau signé et daté comme la Sainte Conversation de Lisbonne (fig. 2) n'est pas mentionné dans son catalogue de 1986, alors qu'il est connu depuis 1972 - ce n'est pas Zampetti l'auteur de sa découverte, mais un rival local, Luigi Dania ${ }^{17}$...

Si le regard de l'historien vasarien est à la source de son écriture, c'est que son attention se porte avant tout vers les formes visuelles. L'attention prêtée par Zampetti à l'histoire des formes est aussi grande que son désintérêt pour tout ce qui en échappe - l'iconographie, le rôle des commanditaires, le contexte culturel. Il a cependant besoin de partir de la biographie de Crivelli pour expliquer l'œuvre, comme chez Vasari. Mais l'absence de vie vasarienne et la rareté des archives sur Crivelli mettent à mal ce postulat. Zampetti est donc obligé de tomber dans le cercle vicieux décrit par Fernando Marias à propos du Greco: on en est réduit à " expliquer l'œuvre d'un peintre à partir de sa vie et, en prenant appui sur cette même œuvre, à construire une biographie permettant à son tour d'éclairer l'œuvre ${ }^{18}$. Ainsi, puisque les figures de Crivelli dénotent "un plasticisme exaspéré, contorsionné " ${ }^{19}$, c'est sans doute qu'il est lui-même "fantaisiste et inspiré " ${ }^{20}$, parcouru par $\mathrm{d}^{\prime}$ " inquiètes visions ". En interprétant psychologiquement l'œuvre de Crivelli, Zampetti la tire vers la modernité - omettant son côté " byzantin ", négligeant son milieu culturel marchisan, sauf pour y voir la cause du déclin de son inspiration, comme Longhi.

Zampetti a ainsi revalorisé la peinture de Crivelli, mais sans réussir à définir positivement, pour lui-même, son style ; et cela parce qu'il est pris dans cette écriture vasarienne qui lui impose une méthodologie et des présupposés qui ne conviennent pas à ce peintre : la vie et l'œuvre, les influences, bref, le schéma vasarien.

\section{L'historien décrit}

Complètement à l'opposé de la démarche zampettienne, le récent livre de Ronald Lightbown ${ }^{21}$ propose un autre genre d'écriture de la monographie: l'écriture panofskyenne, du nom d'Erwin Panofsky, auteur des modèles du genre ${ }^{22}$. Cette écriture fait correspondre l'œuvre et son contexte et délimite comme ligne de coïncidence l'iconographie des œuvres. Dès lors, la question de l'auteur et de son style passe au second plan. À la rigueur, Lightbown aurait pu écrire une monographie sur n'importe quel crivellesque. Il affirme en effet que l'évolution du style de Crivelli n'est pas le plus important dans son œuvre (p. 1). Aussi se concentre-t-il exclusivement sur le sujet de ses tableaux: les saints représentés, les détails symboliques. Le regard de Lightbown n'est pas celui d'un attributionniste guettant le détail stylistique révélateur, mais d'un enquêteur qui cherche les indices d'un message caché dans l'œuvre par ses commanditaires et adressé à ses spectateurs d'origine. Cette différence se manifeste très bien dans la mise en page des deux livres : chez Zampetti, le texte n'est qu'une introduction au catalogue des images, ou des notices informatives. Chez Lightbown, l'image est insérée dans le texte et s'y trouve noyée. Chez Zampetti, les images sont des monuments, le texte, leur légende ; chez Lightbown, les images sont des documents, illustrant le texte.

La monographie de Lightbown possède néanmoins un point commun avec celle de Zampetti : la transparence de son choix d'écriture. Une simple lecture du sommaire nous en montre un effet. Après un chapitre sur la formation de Crivelli à Padoue, Lightbown place pas moins de quarante pages, qui encadrent historiquement la peinture de Crivelli. On saura tout du contexte politique, de l'atmosphère religieuse, des situations culturelles et économique de la Marche d'Ancône au $\mathrm{XV}^{\mathrm{e}}$ siècle. Beaucoup d'informations données n'ont aucun rapport avec Crivelli; mais elles donnent au lecteur un sentiment de réalisme historique, à la manière des longues descriptions des romans du $\mathrm{XIX}^{\mathrm{e}}$ siècle qui visent à rendre l'histoire qui sera racontée la plus vraisemblable possible.

Historien par le rôle déterminant qu'il attribue au contexte, Lightbown l'est aussi par son ton. Je parle ici de l'histoire par opposition au discours, tels que les a définis Benvéniste : l'histoire est un récit dont l'énonciateur se met en retrait, comme si les faits se racontaient d'eux-mêmes; dans le discours, l'énonciateur se met au premier plan ${ }^{23}$. Là où Zampetti fait montre d'une certaine sympathie pour l'artiste dont il a étudié l'œuvre pendant toute sa carrière, Lightbown décrit les tableaux et 
3. Carlo Crivelli, Saint Thomas d'Aquin, 1494, Londres, $\mathrm{Na}$ tional Gallery, (détail).

4. Carlo Crivelli, Saint Thomas d'Aquin, 1494, détail avec la silhouette dans la porte. identifie leurs sujets dans la langue la plus neutre, la plus impersonnelle possible. Cette austérité de l'expression véhicule l'idée de la scientificité de son propos. Le livre entier ne semble être qu'une longue des-

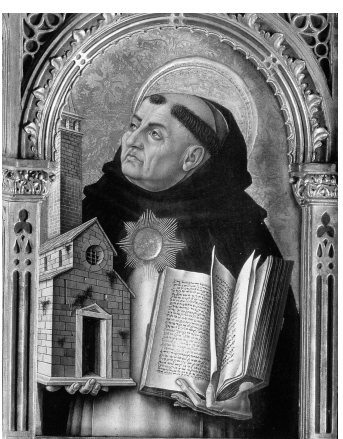
cription : rarement une interprétation vient apporter le trouble d'une opinion personnelle dans ce flux descriptif.

D'ailleurs, Lightbown ne semble rien oublier de décrire : pas un tableau, pas un centimètre carré de peinture ne paraît échapper à la loupe de l'iconographe. L'écriture iconographique suppose en effet que l'image soit entièrement " traduite " en mots, car l'identification correcte du sujet doit pouvoir prendre en compte tous les éléments de l'œuvre. C'est la leçon de Panofsky, théorisant sa méthode : la signification d'une œuvre d'art apparaît une fois que l'on s'en est fait une représentation verbale, quand le tableau est devenu un texte ${ }^{24}$. Ce qui échappe à ce processus de formulation est " insignifiant", au sens où cela ne signifie rien (puisqu'on ne peut pas le lire) et au sens où cela ne compte pour rien (puisque ce qui est important dans une œuvre d'art est ce qui est signifiant).

Appliquant scrupuleusement cette méthode, Lightbown décrit et traduit chaque attribut de saint, chaque élément de vêtement, chaque couleur, chaque ornement : tout chez Crivelli semble être symbolique. Mais on peut se demander si son écriture iconographique ne le conduit pas à interpréter systématiquement en termes de symboles des éléments qui pourraient mériter une interprétation différente (formelle, par exemple) et à omettre de ses descriptions des détails non symboliques et pourtant riches de sens. Ainsi, Lightbown a oublié de mentionner un petit détail dans le Saint Thomas d'Aquin (fig. 3) du polyptyque de 1476, aujourd'hui à Londres (National Gallery) : dans l'église miniature portée par le saint, on devine, dans l'embrasure de la porte, une silhouette non identifiable (fig. 4$)^{25}$. Sa présence est singulière et quasiment invisible ; détail imperceptible, il ne suscite pas l'intérêt de l'iconographe qui ne peut pas identifier la figure, lui accorder une quelconque signification symbolique. Pourtant, dans son écart à la tradition, dans sa discrétion même,

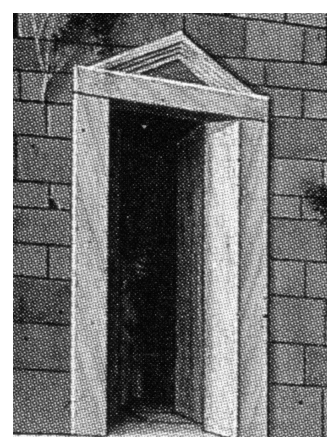

ce détail me semble comme une trace de la présence du peintre dans son œuvre, comme une signature cachée. Lightbown va jusqu'à imaginer que Crivelli s'est constitué une sorte de manuel personnel d'iconographie végétale et florale, puisant çà et là dans les sources textuelles avant de peindre ses fleurs et ses concombres : iconographe avant d'être peintre (p. 11).

Les monographies de Zampetti et de Lightbown sont donc exemplaires : elles se complètent, l'une étant le revers de l'autre ; et elles présentent, en toute naïveté théorique, deux façons d'écrire l'histoire de l'art qui possèdent chacune leurs mérites et leurs limites. D'un côté, Zampetti reconnaît en Crivelli un grand peintre, pourvu qu'on y voie un disciple de Bellini; de l'autre, Lightbown voit dans l'œuvre de Crivelli un document historique éloquent - stylistiquement sans intérêt. Est-ce à dire qu'une monographie sur Crivelli, rendant justice à sa personnalité artistique, est impossible, comme le disait Berenson, avec une pointe de regret $^{26}$ ? Pas forcément. Mais cela nécessite peutêtre de rompre avec le modèle vasarien, d'inventer d'autres généalogies, en s'inspirant, par exemple, des pistes lancées par Federico Zeri, dans Renaissance et pseudo-Renaissance, qui admire en Crivelli "la rage fantastique du dessin " - à condition bien sûr de ne pas voir dans cet autre Quattrocento qu'une simple "traduction dévoyée " de la florentine " Renaissance authentique " ${ }^{27}$.

1. Voir Matthias Waschek éd., Les «Vies " d'artistes, (colloque, Paris, Musée du Louvre, octobre 1993), Paris, 1996.

2. Luigi Lanzi, Storia pittorica della Italia (1793), Florence, $5^{\mathrm{e}}$ éd., 1834, t. III, p. 21-22.

3. Raimond Van Marle, The development of italian schools of painting, t. XVIII, La Haye, 1936, p. 1-68; Adolfo Venturi, Storia dell'arte italiana, VII, "La pittura del Quattrocento ", parte III, Milan, 1914, p. 346-94.

4. Roberto Longhi, " Crivelli e Mantegna : due mostre interferenti e la cultura artistica nel 1961 ", dans Paragone, n 145 , 1962, p. 7-21.

5. George McNeill Rushforth, Carlo Crivelli, Londres, 1900. 
6. Voir Flaminia Gennari Santori, « 'They will form such an ornament for our gallery' : la National Gallery e la pittura di Carlo Crivelli (1856-1868)", dans A. C. Tommasi éd., Giovanni Battista Cavalcaselle conoscitore e conservatore, Venise, 1998, p. 291-312.

7. Voir Gillian Naylor, The Arts and Crafts Movement. A study of its sources, ideals and influence on design theory, Londres, 1990.

8. Franz Drey, Carlo Crivelli und seiner Schüle, Munich, 1927.

9. La majorité des textes publiés en Italie sur Crivelli ne sont que des notices de catalogue résumant les positions de Zampetti, comme si celui-ci avait fourni les clés d'interprétation définitives : par exemple Anna Bovero, Carlo Crivelli, Milan, 1961 ; Emanuela Daffra, "Carlo Crivelli », dans A. De Marchi éd., Pittori a Camerino nel Quattrocento, Milan, 2002, p. 420-31, qui reconnaît partir des "raisonnements fondamentaux " de Zampetti.

10. Pour les plus récentes : Norman Land, "Giotto's fly, Cimabue's gesture, and a Madonna and Child by Carlo Crivelli ", dans Source, XV, n4, 1996, p. 11-15 ; Thomas Golsenne, "L'Annonciation de Carlo Crivelli et le problème de l'ornement ", dans Studiolo. Revue d'histoire de l'art de l'Académie de France à Rome, n², 2002, p. 149-76 ; Bernard Aikema, «Il gusto del paradiso e la persona del pittore. Frutti, firme e altri particolari di Carlo Crivelli », dans I. Chiappini di Sorio, L. De Rossi éd., Venezia, le Marche e la civiltà adriatica, Venise, 2003, p. 194-199.

11. Pittura veneziana nelle Marche, Bergame, 1950 et Carlo Crivelli e i crivelleschi, Venise, 1961.

12. Pietro Zampetti, Carlo Crivelli, Milan, 1961, et Carlo Crivelli, Florence, 1986 (la seconde étant essentiellement une reprise et une mise à jour de la première).

13. Zampetti, 1961, cité n. 12, p. 26-28.

14. Idée formulée franchement par Andrea De Marchi, 2002, cité n. 9, p. 86 : " On préfèrele jeune Crivelli, celui des polyptyques de Massa Fermana, de Montfiore dell'Aso et d'Ascoli Piceno, parce qu'ils ressemblent plus aux œuvres initiales de Giovanni Bellini [...] ".

15. Tandis que la langue est "un corps de prescriptions et d'habitudes, commun à tous les écrivains d'une époque ", et le style, le "secret " de l'écrivain, une " nécessité " inconsciente qui puise dans ses "profondeurs mythiques ", Roland Barthes, Le degré zéro de l'écriture (1953), repris dans Euvres complètes, Paris, 2002, t. I, p. 177-79.

16. Zampetti, 1961, cité n.12, p. 27. Attribution et jugement qui n'ont été jamais remis en doute.

17. Luigi Dania, «Un Crivelli ritrovato : la tavola dell'Annunziata di Ascoli Piceno ", dans S. Bracci éd., Il culto e l'immagine. San Giacomo della Marca (1393-1476) nell'iconografia Marchigiana, Milan, 1998, p. 71-75. L'attribution de Dania se fonde sur des archives et sur des descriptions de l'œuvre in situ au XVIII ${ }^{\mathrm{e}} \mathrm{s}$.

18. Fernando Marias, Greco. Biographie d'un peintre extravagant, Paris, 1997, p. 11.

19. Zampetti, 1961, cité n. 12, p. 26.

20. Zampetti, 1961, cité n. 12, p. 9.

21. Ronald Lightbown, Carlo Crivelli, New York/Londres, 2004.
22. Erwin Panofsky, La vie et l'art d'Albrecht Dürer (Princeton, 1943), Paris, 1987, et surtout Le Titien. Questions d'iconographie (Princeton, 1964), Paris, 2004.

23. Émile Benveniste, Problèmes de linguistique générale, I, Paris, 1976, p. 238-42.

24. Erwin Panofsky, "Contribution au problème de la description d'œuvres appartenant aux arts plastiques et à celui de l'interprétation de leur contenu " (1927), dans La perspective comme forme symbolique et autres essais, Paris, 1975 , p. 235-55.

25. Détail publié dans Bovero, 1975, cité n. 9, et analysé par John Watkins, "Untricking the eye : the uncomfortable legacy of Carlo Crivelli ", dans Art international, n5, 1988, p. 48-58, un des seuls articles absents de la bibliographie de Lightbown.

26. Bernard Berenson, Italian painters of the Renaissance, Londres, 1930, t. I, p. 13.

27. Federico Zeri, Renaissance et Pseudo-Renaissance (Turin, 1985), Paris, 1988, p. 117-18 sur Crivelli, et p. 126 sur la " zone restreinte dans laquelle [le rationalisme de la Renaissance] a fleuri, parmi les déviations et les traductions dévoyées et dans un humus mental et culturel peu enclin à en comprendre le sens véritable ".

Thomas Golsenne, École nationale supérieure des beaux-arts, thomas.golsenne@free.fr

\section{La monographie en musicologie : compositeurs français des XVII ${ }^{\mathrm{e}}$ et $\mathrm{XVIII}^{\mathrm{e}}$ siècles}

\section{Catherine Cessac}

Depuis quelques décennies, la musique française des $\mathrm{XVII}^{\mathrm{e}}$ et $\mathrm{XVIII}^{\mathrm{e}}$ siècles rencontre une audience croissante auprès des interprètes qui exhument régulièrement des partitions oubliées et auprès du public qui se presse nombreux et enthousiaste au concert. La saison d'automne du Centre de Musique Baroque de Versailles dans les lieux prestigieux du château de Versailles ou les festivals spécialisés aux quatre coins de la France rencontrent à cet égard un succès certain. Chaque concert ou presque est l'occasion de découvrir des œuvres inédites et même des compositeurs dont aucune note de musique n'avait résonné depuis des siècles. Dans ce contexte, les musiciens ne se contentent pas de jouer leur rôle d'interprète, mais 